ИЗВЕСТИЯ АКАДЕМИИ НАУК ЭСТОНСКОН ССР. ТОМ ХІV СЕРИЯ ФИЗИКО-МАТЕМАТИЧЕСКИХ И ТЕХНИЧЕСКИХ НАУК. 1965, № 2

\title{
P. ЮРГЕНСOH
}

\section{ОБ ОЦЕНКЕ ПОГРЕШНОСТИ НЕКОТОРЫХ КОНЕЧНО- РАЗНОСТНЫХ МЕТОДОВ ПРИ РЕШЕНИИ ОБЫКНОВЕННЫХ ЛИНЕЙЫХ ДИФФЕРЕНЦИАЛЬНЫХ УРАВНЕНИИ}

Рассматривается применение общих оценок погрешности метода конечных разностей, полученных в статье [3], к конкретным конечноразностным методам - к обыкновенным (пп. 1 и 4), к улучшенным (п. 2) и к многоточечным (п. 3) конечноразностным методам. Особое внимание уделяется нахождению дискретной функции Грина, входящей в оценки погрешности.

\section{1. Обыкновенный метод конечных разностей}

Обыкновенным разностным методом при решении краевой задачи *

$$
\begin{gathered}
x^{(n)}+\sum_{k=0}^{n-1} p_{k}(s) x^{(k)}=f(s), \\
U_{j}(x) \equiv \sum_{k=0}^{n-1}\left[a_{j k} x^{(k)}(a)+b_{j k} x^{(k)}(b)\right]=c_{j} \quad(j=1,2, \ldots, n),
\end{gathered}
$$

где функции $p_{k}(s), f(s)$ являются непрерывными на отрезке $a \leqslant s \leqslant b$, обычно называют (см., напр., $[1,2])$ такой разностный метод, при котором производные $k$-го порядка из дифференциального уравнения (1) в точках $s_{i}$ аппроксимируются с помощью центральных разностей $k$-го порядка, т. е. используют следующие формулы численного дифференцирования

$$
x^{(k)}\left(s_{i}\right)=\frac{\Delta^{k} x\left({ }^{s}{ }_{i-\frac{k}{2}}\right)}{h^{k}}-R_{i}^{(k)},
$$

где $R_{i}^{(k)}$ - остаточный член соответствующей формулы численного дифференцирования. При этом $R_{i}^{(0)}=0$.

Согласно этому определению, система обыкновєнного разностного метода, применительно к краевой задаче $\{(1),(2)\}$, при четном $n$ будет следующая:

\footnotetext{
- Существование единственного решения $x^{*}(s)$ задачи $\{(1),(2)\}$ предполагается.
} 


$$
\begin{aligned}
& \frac{\Delta^{n} x_{i}-\frac{n}{2}}{h^{n}}+\sum_{k=0}^{n-1} p_{k i} \frac{\Delta^{k} x_{i}-\frac{k}{2}}{h^{k}}=f_{i} \quad(i=\vec{p}, \vec{p}+1, \ldots, \bar{q}), \\
& V_{j}\left(x_{v}\right) \equiv \sum_{k=0}^{n-1}\left[a_{i k} \frac{D_{0}^{(k)}\left(x_{v}\right)}{h^{k}}+b_{j k} \frac{D_{m}^{(k)}\left(x_{v}\right)}{h^{k}}\right]=c_{i} \quad(j=1,2, \ldots, n),
\end{aligned}
$$

где $p_{k i}=p_{k}\left(s_{i}\right), f_{i}=f\left(s_{i}\right), \quad s_{i}=a+i h, \quad h=\frac{b-a}{m}$,

$m-$ число отрезков, на которое разбит отрезок $a \leqslant x \leqslant b$, a $\frac{D_{0}\left(x_{v}\right)}{h^{k}}$ и $\frac{D_{m}\left(x_{v}\right)}{h^{k}}-$ некоторые линейные выражения относительно $x_{v}$, аппроксимирующие производные $x^{(k)}(a)$ и $x^{(k)}(b)$. Остаточные члены соответствующих формул численного дифференцирования обозначим через $R_{0}^{(k)}$ и $R_{m}^{(k)}\left(R_{0}^{(0)}=R_{m}^{(0)}=0\right)$.

Числа $\bar{p}, \bar{q}$ и функции $D_{0}^{(k)}, D_{m}^{(k)}$ нужно выбирать таким образом, чтобы число неизвестных и уравнений в системе $\{(3),(4)\}$ совпадало.

Погрешность метода $\{(3),(4)\}$ изучалась в статье [4].

Разностная система в случае уравнений нечетного порядка несколько отличается от системы $\{(3)$, (4) $\}$. Причина этого в следующем.

Если использовать для аппроксимации производных $x^{(k)}\left(s_{i}\right)$ из дифференциального уравнения только симметричные разностные соотношения $\frac{1}{h^{k}} \Delta^{k} x_{i-\frac{k}{2}}$, то в случае уравнений нечетного порядка число уравнений в разностной системе будет, по крайней мере, на единицу меньше, чем число неизвестных.

Для устранения этого недостатка к системе присоединяют еще одно уравнение

$$
L_{i}\left(x_{v}\right) \equiv \frac{D_{j}^{(n)}\left(x_{v}\right)}{h^{n}}+\sum_{k=0}^{n-1} p_{k i} \frac{D_{j}^{(k)}\left(x_{v}\right)}{h^{k}}=f_{j} \quad(j=\bar{p}-1 \text { или } j=\bar{q}+1),
$$

полученное из дифференциального уравнения путем аппроксимации производных в точках $s=s_{\bar{p}-1}$ или $s=s_{\bar{q}+1}$ некоторыми несимметричными разностными соотношениями, пользуясь формулами численного дифференцирования $\left(R_{j}^{(0)}=0\right)$

$$
x^{(k)}\left(s_{i}\right)=\frac{D_{j}^{(k)}\left(x\left(s_{v}\right)\right)}{h^{k}}-R_{j}^{(k)} .
$$

Приведем оценки погрешности метода $\{(3)$, (4), (5) $\}$, получаемые на основе общих результатов статьи [3].

Согласно $\left[{ }^{3}\right]$, при оценке погрешности этого метода используются дискретная функция Грина $G=\left(g_{i k}\right)$ (см. $\left.\left[^{3}\right]\right)$ системы

$$
\Delta^{n} u_{i-\frac{n}{2}}=0 \quad(i=\bar{p}, \bar{p}+1, \ldots, \bar{q})
$$

при условия 


$$
\begin{array}{ll}
V_{j}\left(u_{v}\right)=0 & (j=1,2, \ldots, n) \\
L_{j}\left(u_{v}\right)=0 & (j=\bar{p}-1 \text { или } j=\bar{q}+1)
\end{array}
$$

и элементы $t_{i}$, удовлетворяющие условиям

$$
\begin{gathered}
\Delta^{n} t_{i-\frac{n}{2}}=0 \quad(i=\vec{p}, \vec{p}+1, \ldots, \vec{q}), \\
V_{i}\left(t_{v}\right)=\sum_{k=1}^{n-1}\left[a_{j k} R_{a}^{(k)}+b_{i k} R_{b}^{(k)}\right] \quad(j=1,2, \ldots, n), \\
L_{j}\left(t_{v}\right)=R_{j}^{(n)}+\sum_{k=1}^{n-1} p_{k i} R_{j}^{(k)} .
\end{gathered}
$$

Введем обозначения

$$
\begin{gathered}
R_{i}=R_{i}^{(n)}+\sum_{k=1}^{n-1} p_{k i} R_{i}^{(k)} \quad(i=\bar{p}, \bar{p}+1, \ldots, \bar{q}), \\
\max _{i=\bar{p}, \bar{p}+1, \ldots \bar{q}}\left|R_{i}-\sum_{k=0}^{n-1} p_{k i} \frac{D_{i}^{(k)}\left(t_{v}\right)}{h^{k}}\right| \leqslant R^{*}, \quad\left|t_{i}\right| \leqslant \tau_{i}, \\
\max _{i=\vec{p}, \bar{p}+1, \ldots, \bar{q}}\left|p_{k i}\right| \leqslant \pi_{k}, \max _{i=\bar{p}, \bar{p}+1, \ldots, \bar{q}} h^{n-k} \sum_{j=\bar{p}}^{\bar{q}}\left|D_{i}^{(k)}\left(g_{v j}\right)\right| \leqslant \mu_{k} \\
(k=0,1, \ldots, k-1) .
\end{gathered}
$$

Т е о рем а 1. Eсли

$$
\mu=\sum_{k=0}^{n-1} \pi_{k} \mu_{k}<1
$$

то система $\{(3),(4)$, (5) $\}$ имеет единственное решение $\left\{x_{i}\right\}$, причем справедливо неравенство

$$
\begin{gathered}
\left|x^{*}\left(s_{i}\right)-x_{i}\right| \leqslant \tau_{i}+h^{n} \sum_{k=p}^{\vec{q}}\left|g_{i k}\right| \frac{R^{*}}{1-\mu} \\
\left(i=\bar{p}-\frac{n-1}{2}, \quad \bar{p}-\frac{n-1}{2}+1, \ldots, \bar{q}+\frac{n-1}{2}, \quad j ; \quad j=\bar{p}-\frac{n-1}{2}-1\right. \text { или } \\
\left.j=\bar{q}+\frac{n-1}{2}+1\right) .
\end{gathered}
$$

Рассмотрим, как найти дискретную функцию Грина системы $\{(6)$, (7), (8) \}.

Линейно-независимыми решениями $u_{i}(i=0,1, \ldots, n)$ уравнений (10) являются

$$
u_{0}=1, u_{1}=i, \ldots, \quad u_{n-1}=i^{n-1}, u_{n}=(-1)^{i} .
$$

Поэтому ищем элементы $g_{i k}$ матрицы $G$ в виде 


$$
g_{i k}=\left\{\begin{array}{lr}
a_{0}+a_{1} i+\ldots+a_{n-1} i^{n-1}+a_{k}(-1)^{i} & \text { при } i \leqslant k \\
b_{0}+b_{1} i+\ldots+b_{n-1} i^{n-1}+b_{n}(-1)^{i} & \text { при } i \geqslant k .
\end{array}\right.
$$

По определению дискретной функции Грина, данной в статье [3], элементы $g_{i k}$ должны удовлетворять условиям:

$$
\begin{aligned}
& \Delta^{n} g_{i-\frac{n}{2}, k}= \begin{cases}0 & \text { при } i \neq k, \\
1 & \text { при } i=k(i, k=\bar{p}, \bar{p}+1, \ldots, \bar{q}),\end{cases} \\
& V_{j}\left(g_{v k}\right)=0 \quad(j=1,2, \ldots, n ; k=\vec{p}, \vec{p}+1, \ldots, \vec{q}), \\
& L_{i}\left(g_{\vee k}\right)=0 \quad(j=\bar{p}-1 \text { или } j=\bar{q}+1 ; k=\bar{p}, \bar{p}+1, \ldots, \bar{q}) .
\end{aligned}
$$

Требование выполнения условий (10) приводит к следующей системе уравнений относительно $c_{j}=b_{j}-a_{j}(j=0,1, \ldots, n)$ :

$$
\begin{gathered}
\sum_{i=0}^{n-1} c_{i}(k-j)^{i}+c_{n}(-1)^{k-j}=0 \quad\left(j=0, \pm 1, \ldots, \pm \frac{n-1}{2}\right), \\
2^{n}(-1)^{k} c_{n}=2 .
\end{gathered}
$$

Эту систему преобразуем к виду

$$
\begin{gathered}
\sum_{i=j}^{n-1} c_{i} \Delta^{j}\left(k-\frac{j}{2}\right)^{i}+c_{n} \Delta^{j}(-1)^{k-\frac{j}{2}}=0 \quad(j=0,1, \ldots, n-1), \\
2^{n}(-1)^{k} c_{n}=2,
\end{gathered}
$$

где

$$
\begin{aligned}
\Delta^{j}\left(k-\frac{j}{2}\right)^{i}=\left\{\begin{array}{cc}
\sum_{\nu=0}^{j}(-1)^{\nu} C_{j}^{\nu}\left(k+\frac{j}{2}-v\right)^{i} & \text { при четном } j, \\
\frac{1}{2} \sum_{\nu=0}^{j}(-1)^{\nu} C_{j}^{\nu}\left[\left(k+\frac{j+1}{2}-v\right)^{i}+\left(k+\frac{j-1}{2}-v\right)^{i}\right]
\end{array}\right. \\
\Delta^{j}(-1)^{k-\frac{j}{2}}=\left\{\begin{array}{rr}
\sum_{\nu=0}^{j}(-1)^{\nu} C_{j}^{\nu}(-1)^{k+\frac{j}{2}-\nu} & \text { при четном } j, \\
\frac{1}{2} \sum_{\nu=0}^{j}(-1)^{\nu} C_{j}^{\nu}\left[(-1)^{k+\frac{j+1}{2}-\nu}+(-1)^{k+\frac{j-1}{2}-\nu}\right] & \text { при нечетном } j .
\end{array}\right.
\end{aligned}
$$

Так как

$$
c_{n}=\frac{(-1)^{k}}{2^{n-1}}, \quad \sum_{\nu=0}^{j}(-1)^{\nu} C_{j}^{\nu}(-1)^{k+\frac{j}{2}-\nu}=2^{j}(-1)^{k+\frac{j}{2}},
$$




$$
\begin{gathered}
\frac{1}{2} \sum_{\nu=0}^{j}(-1)^{\nu} C_{j}^{\nu}\left[(-1)^{k+\frac{j+1}{2}-\nu}+(-1)^{k+\frac{j-1}{2}-\nu}\right]= \\
=\frac{1}{2}(-1)^{k+\frac{j}{2}}\left[(-1)^{-\frac{1}{2}}+(-1)^{\frac{1}{2}}\right]=0
\end{gathered}
$$

то можем последнюю систему переписать в виде

$$
c_{n}=\frac{(-1)^{k}}{2^{n-1}}, \quad \sum_{i=j}^{n-1} c_{i} \Delta^{j}\left(k-\frac{j}{2}\right)^{i}= \begin{cases}2^{j-n+1}(-1)^{\frac{j+2}{2}} \text { при } j=0,2, \ldots, n-1, \\ 0 & \text { при } j=1,3, \ldots, n-2 .\end{cases}
$$

Присоединяя к этой системе уравнения, получаемые из требований (11), (12), получим систему для определения $a_{j}$ и $b_{j}(j=0,1, \ldots, n)$.

При ме р 1. Элементы $g_{i k}$ системы*

$$
\begin{gathered}
u_{i+1}-u_{i-1}=0(i=1,2, \ldots, m-1), \\
u_{0}=0 \\
\frac{u_{1}-u_{0}}{h}+p_{0} u_{0}=0
\end{gathered}
$$

имеют вид

$$
g_{i k}= \begin{cases}0 & \text { при } i \leqslant k \\ 1+(-1)^{i+k+1} & \text { при } i \geqslant k\end{cases}
$$

Легко найти

$$
\mu_{0}=\left\{\begin{array}{cc}
b-a-h & \text { при четном } m, \\
b-a-2 h & \text { при нечетном } m .
\end{array}\right.
$$

Пр и мер 2. Для оценки погрешности конечно-разностного метода

$$
\begin{gathered}
\frac{x_{i+2}-2 x_{i+1}+2 x_{i-1}-x_{i-2}}{2 h^{3}}+p_{1 i} \frac{x_{i+1}-x_{i-1}}{2 h}+p_{0 i} x_{i}=f_{i} \quad(i=1,2, \ldots, m-1), \\
x_{0}=c_{0}, \quad \frac{x_{1}-x_{-1}}{2 h}=c_{1}, \frac{x_{m+1}-2 x_{m}+x_{m-1}}{h^{2}}=c_{2}, \\
\frac{x_{2}-3 x_{1}+3 x_{0}-x_{-1}}{h^{3}}+p_{10} \frac{x_{1}-x_{-1}}{2 h}+p_{00} x_{0}=f_{0}
\end{gathered}
$$

* Элементы $g_{i k}$ этой системы нужны при оценке погрешности метода

$$
\frac{x_{i+1}-x_{i-1}}{2 h}+p_{i} x_{i}=f_{i}, x_{0}=0, \frac{x_{1}-x_{0}}{h}+p_{0} x_{0}=f_{0}
$$

решения краевой задачи

$$
x^{\prime}+p(s) x=f(s), x(a)=c_{0}
$$


при решении задачи

$$
x^{\prime \prime \prime}+p_{1}(s) x^{\prime}+p_{0}(s) x=f(s),
$$

$$
x(a)=c_{0}, x^{\prime}(a)=c_{1}, x^{\prime \prime}(b)=c_{2}
$$

нужно знать элементы $g_{i k}$ системы

$$
\begin{gathered}
u_{i+2}-2 u_{i+1}+2 u_{i-1}-u_{i-2}=0 \quad(i=1,2, \ldots, m-1), \\
u_{0}=0, \frac{u_{1}-u_{-1}}{2 h}=0, \quad \frac{u_{m i+1}-2 u_{m}+u_{m-1}}{h^{2}}=0, \\
\frac{u_{2}-3 u_{1}+3 u_{0}-u_{-1}}{h^{3}}+p_{10} \frac{u_{1}-u_{-1}}{2 h}+p_{00} u_{0}=0 .
\end{gathered}
$$

Пользуясь изложенным выше методом, найдем

$$
g_{i k}=\frac{1}{2} \cdot\left\{\begin{array}{l}
{\left[(-1)^{m+k}-1\right] i^{2} \quad \text { при } i \leqslant k,} \\
(-1)^{m+k} i^{2}-2 k i+k^{2}-\frac{1}{2}(-1)^{k+i} \quad \text { при } i \geqslant k .
\end{array}\right.
$$

\section{2. Улучшенный метод конечных разностей}

Улучшенным методом конечных разностей называют * (см., напр., [']) такой разностный метод, при котором производные $x^{(k)}\left(s_{i}\right)$ заменяют не простейшими разностными соотношениями $\frac{1}{h^{k}} \Delta^{k} x_{i}-\frac{k}{2}$ (как в случае обыкновенного разностного метода), а более сложными разностными выражениями, которые точнее аппроксимируют производные, чем простейшие разностные соотношения.

Так, для замены производной $x^{\prime \prime}\left(s_{i}\right)$ разностным выражением при обыкновенном разностном методе используется формула

$$
x^{\prime \prime}\left(s_{i}\right)=\frac{x\left(s_{i+1}\right)-2 x\left(s_{i}\right)+x\left(s_{i-1}\right)}{h^{2}}-\frac{h^{2}}{12} x^{\mathrm{IV}}\left(\bar{\xi}_{i}\right) \quad\left(s_{i-1} \leqslant \bar{\xi}_{i} \leqslant s_{i+1}\right),
$$

а при улучшенном разностном методе, например, формула

$$
\begin{gathered}
x^{\prime \prime}\left(s_{i}\right)=\frac{-x\left(s_{i+2}\right)+16 x\left(s_{i+1}\right)-30 x\left(s_{i}\right)+16 x\left(s_{i-1}\right)-x\left(s_{i-2}\right)}{12 h^{2}}+\frac{h^{4}}{90} x^{\mathrm{VI}}\left(\xi_{i}\right) \\
\left(s_{i-2} \leqslant \xi_{i} \leqslant s_{i+2}\right) .
\end{gathered}
$$

Улучшенный разностный метод исследуем на примере краевой задачи для дифференциального уравнения второго порядка

$$
\left.\begin{array}{l}
x^{\prime \prime}+p(s) x=f(s) \\
x(a)=x(b)=0
\end{array}\right\}
$$

* Некоторые авторы, например Л. Коллац [], называют этот метод методом конечных разностей повышенной точности. 
Используя формулу (13), составим систему

$$
\left.\begin{array}{l}
\frac{-x_{i+2}+16 x_{i+1}-30 x_{i}+16 x_{i-1}-x_{i-2}}{12 h^{2}}+p_{i} x_{i}=f_{i} \\
(i=1,2, \ldots, m-1), \quad x_{0}=x_{m}=0 .
\end{array}\right\}
$$

Чтобы исключить неизвестные $x_{-1}, x_{m+1}$, присоединяем для граничных точек $s_{0}, s_{m}$ обыкновенные разностные уравнения

$$
\left.\begin{array}{c}
\frac{x_{1}-2 x_{0}+x_{-1}}{h^{2}}+p_{0} x_{0}=f_{0}, \\
-2 x_{m}+x_{m-1}+p_{m} x_{m}=f_{m} .
\end{array}\right\}
$$

Для оценки погрешности метода $\{(15),(16)\}$ по оценкам статьи [ $\left.{ }^{3}\right]$ нужно найти дискретную функцию Грина $G=\left(g_{i k}\right)$ системы

$$
\begin{aligned}
& \frac{1}{12}\left(-u_{i+2}+16 u_{i+1}-30 u_{i}+16 u_{i-1}-u_{i-2}\right)=0 \\
&(i=1,2, \ldots, m-1)
\end{aligned}
$$

ппри условиях

$$
\begin{gathered}
u_{0}=u_{m}=0, \frac{u_{1}-2 u_{0}+u_{-1}}{h^{2}}+p_{0} u_{0}=0, \\
\frac{u_{m+1}-2 u_{m}+u_{m-1}}{h^{2}}+p_{m} u_{m}=0
\end{gathered}
$$

и элементы $t_{i}$, удовлетворяющие условиям

$$
\begin{gathered}
\frac{1}{12}\left(-t_{i+2}+16 t_{i+1}-30 t_{i}+16 t_{i-1}-t_{i-2}\right)=0 \\
\quad(i=1,2, \ldots, m-1), \\
t_{0}=t_{m}=0 \quad \frac{t_{1}-2 t_{0}+t_{-1}}{h^{2}}+p_{0} t_{0}=\frac{h^{2}}{12} x^{\mathrm{IV}}\left(\bar{\xi}_{0}\right), \\
\frac{t_{m+1}-2 t_{m}+t_{m-1}}{h^{2}}+p_{m} t_{m}=\frac{h^{2}}{12} x^{\mathrm{IV}}\left(\bar{\xi}_{m}\right) .
\end{gathered}
$$

Так как линейно-независимыми решениями уравнения (17) являются

$$
u_{0}=1, u_{1}=i, u_{2}=(7+4 \sqrt{3})^{i}, u_{3}=(7-4 \sqrt{3})^{i},
$$

то $g_{i k}$ ищем в виде

$g_{\text {ik }}= \begin{cases}a_{0}+a_{1} i+a_{2}(7+4 \sqrt{3})^{i}+a_{3}(7-4 \sqrt{3})^{i} & \text { при } i \leqslant k, \\ b_{0}+b_{1} i+b_{2}(7+4 \sqrt{3})^{i}+b_{3}(7-4 \sqrt{3})^{i} & \text { при } i \geqslant k\end{cases}$ 
Элементы $g_{i k}$ должны удовлетворять условиям

$-g_{i+2, k}+16 g_{i+1, k}-30 g_{i k}+16 g_{i-1, k}-g_{i-2, k}=\left\{\begin{array}{c}0 \text { при } i \neq k, \\ 12 \text { при } i=k\end{array}\right.$

$(i, k=1,2, \ldots, m-1)$,

$g_{0 k}=g_{m k}=0, \quad g_{1 k}-2 g_{0 k}+g_{-1, k}=0$,

$\left.\left.g_{m+1, k}-2 g_{m k}+g_{m-1, k}=0 \quad i k=1,2, \ldots, m-1\right).\right\}$

Из требований (20) получим уравнения $\left(c_{j}=b_{j}-a_{j}\right)$

$$
\begin{aligned}
& c_{0}+k c_{1}+(7+4 \sqrt{3})^{k} c_{2}+(7-4 \sqrt{3})^{k} c_{3}=0, \\
& c_{0}+(k+1) c_{1}+(7+4 \sqrt{3})^{k+1} c_{2}+(7-4 \sqrt{3})^{k+1} c_{3}=0, \\
& c_{0}+(k-1) c_{1}+(7+4 \sqrt{3})^{k-1} c_{2}+(7-4 \sqrt{3})^{k-1} c_{3}=0, \\
& c_{1}+8 \sqrt{3}(7+4 \sqrt{3})^{k} c_{2}-8 \sqrt{3}(7-4 \sqrt{3})^{k} c_{3}=12,
\end{aligned}
$$

च из требований (21) - уравнения

$$
\begin{aligned}
& a_{0}+a_{2}+a_{3}=0 \\
& b_{0}+m b_{1}+(7+4 \sqrt{3})^{m} b_{2}+(7-4 \sqrt{3})^{m} b_{3}=0 \\
& a_{2}+a_{3}=0 \\
& (7+4 \sqrt{3})^{m} b_{2}+(7-4 \sqrt{3})^{m} b_{3}=0
\end{aligned}
$$

Решая систему $\{(22),(23)\}$, получим

$$
g_{i k}=\left\{\begin{array}{l}
\frac{i(k-m)}{m}+\frac{\left[(7+4 \sqrt{3})^{m-k}-(7-4 \sqrt{3})^{m-k}\right]\left[(7+4 \sqrt{3})^{i}-(7-4 \sqrt{3})^{i}\right]}{8 \sqrt{3}\left[(7+4 \sqrt{3})^{m}-(7-4 \sqrt{3})^{m}\right] \quad \text { при } \mathrm{i} \leqslant k,} \\
\frac{k(i-m)}{m}+\frac{\left[(7+4 \sqrt{3})^{m-i}-(7-4 \sqrt{3})^{m-i}\right]\left[(7+4 \sqrt{3})^{k}-(7-4 \sqrt{3})^{k}\right]}{8 \sqrt{3}\left[(7+4 \sqrt{3})^{m}-(7-4 \sqrt{3})^{m}\right] \quad \text { при } i \geqslant k .}
\end{array}\right.
$$

Пользоваться элементами $g_{i k}$ для оценки погрешности в таком общем виде, конечно, трудно. Для конкретных $m$ элементы $g_{i k}$ легко вычисляемы.

Элементы $t_{i}$ представляются в виде

$$
t_{i}=e_{0}+e_{1} i+e_{2}(7+4 \sqrt{3})^{i}+e_{3}(7-4 \sqrt{3})^{i} \quad(i=-1,0,1, \ldots, m+1) .
$$

Неизвестные коэффициенты $e_{j}$ определяются из системы

$$
\begin{aligned}
& e_{0}+e_{2}+e_{3}=0, \\
& e_{0}+m e_{1}+(7+4 \sqrt{3})^{m} e_{2}+(7-4 \sqrt{3})^{m} e_{3}=0, \\
& 12 e_{2}+12 e_{3}=\frac{h^{4}}{12} x^{*} \mathrm{IV}\left(\bar{\xi}_{0}\right), \\
& 12(7+4 \sqrt{3})^{m} e_{2}+12(7-4 \sqrt{3})^{m} e_{3}=\frac{h^{4}}{12} x^{* \mathrm{IV}}\left(\bar{\xi}_{m}\right) .
\end{aligned}
$$


Решая эту систему, найдем

$$
\begin{aligned}
t_{i}= & \frac{h^{4}}{144} x^{\mathrm{IV}}\left(\bar{\xi}_{0}\right)\left[\frac{(7+4 \sqrt{3})^{m-i}-(7-4 \sqrt{3})^{m-i}}{(\bar{i}+4 \sqrt{3})^{m}-(7-4 \sqrt{3})^{m}}-\frac{m-i}{m}\right]+ \\
& +\frac{h^{4}}{144} x^{\mathrm{IV}}\left(\bar{\xi}_{m}\right)\left[\frac{(\bar{i}+4 \sqrt{3})^{i}-(7-4 \sqrt{3})^{i}}{(\bar{i}+4 \sqrt{3})^{m}-(7-4 \sqrt{3})^{m}}-\frac{i}{m}\right] .
\end{aligned}
$$

Теперь мы можем найти оценки погрешности разностного метода $\{(15),(16)\}$. На основании результатов статьи $\left.{ }^{3}\right]$ имеем

$$
\begin{array}{r}
\left|x^{*}\left(s_{i}\right)-x_{i}\right| \leqslant \tau_{i}+h^{2} \sum_{k=1}^{m-1}\left|g_{i k}\right| \frac{R^{*}}{1-\pi_{0} \mu_{0}} \\
(i=-1,0,1, \ldots, m+1),
\end{array}
$$

где

$$
\begin{aligned}
& \left|t_{i}\right| \leqslant \tau_{i}, \quad \max _{i=1,2, \ldots, m-1}\left|p_{i}\right| \leqslant \pi_{0}, \max _{i=1,2, \ldots, m-1} \sum_{k=1}^{m-1}\left|g_{i k}\right| \leqslant \mu_{0} \\
& \max _{i=1,2, \ldots, m-1}\left|\frac{h^{4}}{90} x^{* \mathrm{VI}}\left(\xi_{i}\right)+p_{i} t_{i}\right| \leqslant R^{*} .
\end{aligned}
$$

В случае улучшенных разностных методов решения дифференциальных уравнений более высокого порядка погрешности оцениваются таким же образом. Элементы $g_{i k}$, как правило, имеют сравнительно сложный вид. Но это не препятствует оценке погрешности, так как можно раз и навсегда составить таблицу для значений $g_{i k}$ при конкретных $m$.

Пр и мер. Для приближенного решения краевой задачи

$$
\begin{gathered}
x^{\prime \prime}+x=-s, \\
x(0)=x(1)=0
\end{gathered}
$$

пользуемся разностным методом $\{(15),(16)\}$ с $m=4$.

Решениями системы $\{(15),(16)\}$ являются: $x_{1} \approx 0,044016 ; \quad x_{2} \approx 0,069758$; $x_{3} \approx 0,060075$.

Чтобы оценить точность этих решений, вычислим

$$
\text { для } m=4 \quad G=\left(\begin{array}{ccc}
\frac{1293}{14 \cdot 97} & \frac{64}{97} & \frac{16 \cdot 339}{7 \cdot 24 \cdot 97} \\
0 & 0 & 0 \\
-\frac{1293}{14 \cdot 97} & -\frac{64}{97} & -\frac{16 \cdot 339}{7 \cdot 24 \cdot 97} \\
-\frac{64}{97} & -\frac{90}{97} & -\frac{64}{97} \\
-\frac{16 \cdot 339}{7 \cdot 24 \cdot 97} & -\frac{64}{97} & -\frac{307 \cdot 16}{56 \cdot 97} \\
0 & 0 & 0 \\
\frac{16 \cdot 339}{7 \cdot 24 \cdot 97} & \frac{64}{97} & \frac{307 \cdot 16}{56 \cdot 97}
\end{array}\right) \text {, }
$$




$$
\sum_{k=1}^{3}\left|g_{i k}\right| \leqslant \begin{cases}0,091 \text { при } i=1 \\ 0,12 & \text { при } i=2 \\ 0,09 & \text { при } i=3\end{cases}
$$

\section{Отсюда}

$$
\max _{i=1,2,3} \sum_{k=1}^{3}\left|g_{i k}\right| \leqslant 0,12=\mu_{0} \quad \text { и } \quad \pi_{0} \mu_{0}=0,12<1 .
$$

\section{Найдем также}

$$
\begin{aligned}
t_{1} & =\frac{411}{18232 \cdot 256 \cdot 144} x \operatorname{IV}\left(\bar{\xi}_{0}\right)-\frac{678}{2716 \cdot 256 \cdot 144} x^{\mathrm{iV}\left(\bar{\xi}_{4}\right),} \\
t_{2} & =-\frac{48}{97 \cdot 256 \cdot 144} x \operatorname{IV}\left(\bar{\xi}_{0}\right)-\frac{48}{97 \cdot 256 \cdot 144} x ! \mathrm{V}\left(\bar{\xi}_{4}\right), \\
t_{3} & =-\frac{2712}{10862 \cdot 256 \cdot 144} x \operatorname{IV}\left(\bar{\xi}_{0}\right)-\frac{1842}{2716 \cdot 256 \cdot 144} x^{\mathrm{IV}\left(\bar{\xi}_{4}\right)} .
\end{aligned}
$$
лучим

Оценим производные $x^{\mathrm{IV}}(s), x \mathrm{VI}(s)$. На основании результатов п. 4 статьи $\left.{ }^{4}\right]$ по-

$$
\begin{aligned}
& \operatorname{niax}_{s_{i-1}}|x(s)| \leqslant 0,1773(i=0,4), \quad \operatorname{miax}|x(s)| \leqslant 0,142 . \\
& s_{i+1} \leqslant s \leqslant s_{3}
\end{aligned}
$$

Следовательно,

$$
\begin{aligned}
& \quad \max |x \operatorname{IV}(s)|=\max _{s_{-1} \leqslant s \leqslant s_{1}}|x(s)+s| \leqslant 0,1773+0,25=0,4273, \\
& s_{-1} \leqslant s \leqslant s_{1} \\
& \max |x \operatorname{IV}(s)|=\max _{s_{3} \leqslant s \leqslant s_{5}}|x(s)+s| \leqslant 0,1773+1,25=1,4273, \\
& s_{3} \leqslant s \leqslant s_{5} \\
& \quad \operatorname{nax}|x \operatorname{IV}(s)|=\max _{s_{i-2} \leqslant s \leqslant s_{i+2}}|x(s)+s| \leqslant 0,1773+1,25=1,4273 \\
& s_{i-2} \leqslant s \leqslant s_{i+2} \\
& (i=1,2,3) .
\end{aligned}
$$

Используя эти оценки, вычислим

$$
\begin{gathered}
\tau_{1}=0,00004, \quad \tau_{2}=0,0004, \quad \tau_{3}=0,00044, \\
R^{*}=0,00054, \frac{R^{*}}{1-\pi_{0} \mu_{0}}=0,00061
\end{gathered}
$$

Оценки для $\left\{x\left(s_{i}\right)-x_{i} \mid(i=1,2,3)\right.$ получим из неравенства

$$
\begin{aligned}
& \left|x\left(s_{1}\right)-x_{1}\right| \leqslant 0,000096, \\
& \left|x\left(s_{2}\right)-x_{2}\right| \leqslant 0,00048 \\
& \left|x\left(s_{3}\right)-x_{3}\right| \leqslant 0,00049 .
\end{aligned}
$$

Действительные погрешности в точках $s_{i}(i=1,2,3)$ следующие:

$$
\begin{aligned}
& x\left(s_{1}\right)-x_{1} \approx-0,000017, \\
& x\left(s_{2}\right)-x_{2} \approx-0,000062, \\
& x\left(s_{3}\right)-x_{3} \approx-0,000093 .
\end{aligned}
$$




\section{3. Многоточечный метод конечных разностей}

Многоточечными конечноразностными методами называют (см., напр., $\left.\left[{ }^{2}\right]\right)$ такие разностные методы, которые основаны на использовании линейных комбинаций значений неизвестной функции $x(s)$ и их производных определенного порядка в соседних точках $s_{i}$, т. е. основаны на использовании выражений вида

$$
\sum_{k=i-v}^{i+v}\left[a_{k}^{(0)} x\left(s_{k}\right)+a_{k}^{(j)} x\left(s_{k}\right)\right] .
$$

Для оценки погрешности многоточечных конечноразностных методов общие оценки статьи [3] непосредственно не применимы. Но, как будет показано ниже, с помощью методики, аналогичной методике, используемой нами в статье [3], можно вывести оценки погрешности и для случая многоточечных методов.

Рассмотрим применение многоточечного конечноразностного метода для приближенного решения краевой задачи

$$
\begin{aligned}
x^{(n)}+p(s) x & =f(s), \\
U_{j}(x) & \equiv \sum_{k=0}^{n-1}\left[a_{j k} x^{(k)}(a)+b_{j k} x^{(k)}(b)\right]=c_{j} \quad(j=1,2, \ldots, n),
\end{aligned}
$$

где функции $p(s)$ и $f(s)$ являются непрерывными.

Пусть $n \leqslant 4$.

Используем следующие многоточечные формулы (см. [], табл. III, Добавления) :

$$
\begin{gathered}
\frac{\Delta^{n} x\left(s_{i-\frac{n}{2}}\right)}{h^{n}}= \\
=\sum_{j=-1}^{1} \frac{\alpha_{i} x^{(n)}\left(s_{i+j}\right)}{\alpha}+R_{i}^{(n)}, \\
\text { где } \alpha=\sum_{j=-1}^{1} \alpha_{j}, \\
\text { а } \alpha_{j} \text { и } R_{i}^{(n)}
\end{gathered}
$$

даны в таблице.

\begin{tabular}{c|c|c|c|c}
\hline$n$ & $\alpha_{-1}$ & $\alpha_{0}$ & $\alpha_{1}$ & $R_{i}^{(n)}$ \\
\hline 1 & 1 & 4 & 1 & $-\frac{h^{4}}{180} x \mathrm{~V}\left(s_{i}\right)+\ldots$ \\
2 & 1 & 10 & 1 & $-\frac{h^{4}}{240} x \mathrm{~V} 1\left(s_{i}\right)+\ldots$ \\
3 & 1 & 2 & 1 & $-\frac{h^{4}}{240} x \mathrm{VIII}\left(s_{i}\right)+\ldots$ \\
4 & 1 & 4 & 1 & $-\frac{h^{4}}{720} x \mathrm{VIII}\left(s_{i}\right)+\ldots$
\end{tabular}

Подставляя в формулы (27) выражения

$$
x^{(n)}\left(s_{i}\right)=-p_{i} x\left(s_{i}\right)+f_{i},
$$

где $p_{i}=p\left(s_{i}\right), f_{i}=f\left(s_{i}\right)$, полученные из уравнения (25), имеем $(\bar{p}, \bar{q}-$ некоторые целые числа; $s_{i}$ - узлы подразделения отрезка $a \leqslant s \leqslant b$ )

$$
\frac{\Delta^{n} x\left(s_{i-\frac{n}{2}}\right)}{h^{n}}+\sum_{j=-1}^{1} \frac{\alpha_{j} p_{i+j} x\left(s_{i+j}\right)}{\alpha}=\sum_{\substack{j=-1 \\(i=\bar{p}, \bar{p}+1, \ldots, \bar{q})}}^{1} \frac{\alpha_{j} f_{i+j}}{\alpha}+R_{i}^{(n)}
$$


Производные в краевых условиях (26) заменяем некоторыми разностными выражениями, используя формулы численного дифференцирования.

$$
x^{(k)}(a)=\frac{D_{a}^{(k)}\left(x\left(s_{\mathrm{v}}\right)\right)}{h^{k}}-R_{a}^{(k)}, \quad x^{(k)}(b)=\frac{D_{b}^{(k)}\left(x\left(s_{\mathrm{v}}\right)\right)}{h^{k}}+R_{b}^{(k)} .
$$

Получим соотношения

$$
\begin{aligned}
V_{j}\left(x\left(s_{\nu}\right)\right) & \equiv \sum_{k=0}^{n-1}\left[a_{j k} \frac{D_{a}^{(k)}\left(x\left(s_{\nu}\right)\right)}{h^{k}}+b_{j k} \frac{D_{b}^{(k)}\left(x\left(s_{v}\right)\right)}{h^{k}}\right]= \\
& =c_{j}+\sum_{k=0}^{n-1}\left[a_{j k} R_{a}^{(k)}+b_{j k} R_{b}^{(k)}\right] \quad(j=1,2, \ldots, n)
\end{aligned}
$$

Равенства (28) и (29) рассмотрим как систему уравнений относительно $x\left(s_{i}\right)$. Крайние неизвестные обозначим через $x\left(s_{p}\right)$ и $x\left(s_{q}\right)$. При $n=1$ и $n=3$ для того, чтобы число уравнений и число неизвестных совпадало, к этой системе присоединяют еще одно уравнение, например, следующее:

$$
\frac{D_{j}^{(n)}\left(x\left(s_{v}\right)\right)}{h^{n}}+p_{j} x\left(s_{j}\right)=f_{j}+R_{j}^{(n)} \quad(j=\vec{p}-1 \text { или } j=\bar{q}+1) .
$$

Последнее уравнение получается из (25) с помощью (несимметричной) формулы численного дифференцирования

$$
x^{(n)}\left(s_{j}\right)=\frac{D_{j}^{(n)}\left(x\left(s_{v}\right)\right)}{h^{n}}-R_{j}^{(n)} .
$$

Отбрасывая в системе $\{(28),(29),(30)\}$ остаточные члены $R_{i}^{(n)}$ $(i=\bar{p}, \bar{p}+1, \ldots, \bar{q}, j), R_{a}^{(k)}, R_{b}^{(k)}$, получим систему многоточечного разностного метода

$$
\begin{gathered}
\frac{\Delta^{n} x_{i-\frac{n}{2}}}{h^{n}}+\sum_{j=-1}^{1} \frac{\alpha_{i} p_{i+j} x_{i+j}}{\alpha}=\sum_{j=-1}^{1} \frac{\alpha_{j} f_{i+j}}{\alpha} \quad(i=\bar{p}, \bar{p}+1, \ldots, \bar{q}), \\
V_{j}\left(x_{v}\right)=c_{j} \quad(j=1,2, \ldots, n), \\
\frac{D_{j}^{(n)}\left(x_{v}\right)}{h^{n}}+p_{j} x_{j}=f_{j} \quad(j=\bar{p}-1 \text { или } j=\bar{q}+1) .
\end{gathered}
$$

Для оценки погрешностей $\varepsilon_{i}=x\left(s_{i}\right)-x_{i}$ вычтем эту систему из системы $\{(28),(29),(30)\}$

$$
\begin{aligned}
& \frac{\Delta^{n} \varepsilon_{i}-\frac{n}{2}}{h^{n}}+\sum_{j=-1}^{1} \frac{\alpha_{j} p_{i+j} \varepsilon_{i+j}}{\alpha}=R_{i}^{(n)} \quad(i=\bar{p}, \bar{p}+1, \ldots, \bar{q}), \\
& V_{j}\left(\varepsilon_{v}\right)=\sum_{k=0}^{n-1}\left[a_{i k} R_{a}^{(k)}+b_{j k} R_{b}^{(k)}\right] \\
& \frac{D_{j}^{(n)}\left(\varepsilon_{\mathrm{v}}\right)}{h^{n}}+p_{j} \varepsilon_{j}=f_{j} \quad(j=1,2, \ldots, n),
\end{aligned}
$$


Таким же образом, как в статье [3], преобразуем последнюю систему в следующую:

$$
\eta_{i}+\sum_{j=-1}^{1} \sum_{k=\bar{p}}^{\vec{q}} \frac{\alpha_{i} p_{i+j} g_{i+j, k}}{\alpha} \eta_{k}=R_{i} \quad(i=\vec{p}, \vec{p}+1, \ldots, \vec{q})
$$

Здесь

$$
R_{i}=-\sum_{j=-1}^{1} \frac{\alpha_{j} p_{i+i}}{\alpha} t_{i+j}+R_{i}^{(n)}
$$

$g_{i k}$ - элементы дискретной функции Грина (см. [3]) системы

$$
\Delta^{n} u_{i-\frac{n}{2}, k}=0 \quad(i=\vec{p}, \bar{p}+1, \ldots, \bar{q})
$$

піри условиях *

$$
\begin{aligned}
V_{i}\left(u_{v}\right)=0 & (j=1,2, \ldots, n), \\
\frac{D_{j}^{(n)}\left(u_{\vee}\right)}{h^{n}}+p_{i} u_{j}=0 & (j=\bar{p}-1 \text { или } j=\bar{q}+1),
\end{aligned}
$$

a $t_{i}$ - линейно-независимые решения системы

$$
\Delta^{n} t_{i-\frac{n}{2}}=0 \quad(i=\vec{p}, \vec{p}+1, \ldots, \vec{q}),
$$

удовлетворяющие условиям

$$
\begin{gathered}
V_{i}\left(t_{\mathrm{v}}\right)=\sum_{k=0}^{n-1}\left[a_{i k} R_{a}^{(k)}+b_{j k} R_{b}^{(k)}\right] \quad(j=1,2, \ldots, n), \\
\frac{D_{j}^{(n)}\left(t_{\mathrm{v}}\right)}{h^{n}}+p_{j} t_{j}=R_{j}^{(n)} \quad(j=\bar{p}-1 \text { или } j=\bar{q}+1) .
\end{gathered}
$$

Имеют место равенства

$$
\left.\begin{array}{l}
\varepsilon_{i}=u_{i}+t_{i}, \\
u_{i}=h^{n} \sum_{k=\bar{p}}^{\bar{q}} g_{i k} \eta_{k} \quad(i=p, p+1, \ldots, q) .
\end{array}\right\}
$$

Рассмотрим систему (31) в пространстве $(\bar{q}-\bar{p}+1)$-мерных векторов и обозначим оценки

$$
\begin{gathered}
\left|t_{i}\right| \leqslant \tau_{i}(i=p, p+1, \ldots, q), \underset{i=\vec{p}, \bar{p}+1, \ldots, \bar{q}}{\max }\left|R_{i}\right| \leqslant R^{*}, \\
\max _{i=\bar{p}, \bar{p}+1, \ldots, \bar{q}} \sum_{k=\bar{p}}^{\vec{q}}\left|\sum_{j=-1}^{1} \frac{\alpha_{j} p_{i+j} g_{i+i, k}}{\alpha}\right| \leqslant \mu .
\end{gathered}
$$

* При $n=2$ и $n=4$ условия (32) и (33) отсутствуют. 
В качестве $\mu$ можно взять, например,

$$
\mu=\max _{i=\bar{p}-1, \bar{p}, \ldots, \bar{q}+1}\left|p_{i}\right| \cdot \max _{i=\bar{p}-1, \bar{p}, \ldots, \bar{q}+1} \sum_{k=\bar{p}}^{\bar{q}}\left|g_{i k}\right| .
$$

Предположим, что $\mu<1$. Тогда можем применить известную теорему Банаха, на основании которой имеет место равенство

$$
\max _{i=\bar{p}, \bar{p}+1, \ldots, \bar{q}}\left|\eta_{i}\right| \leqslant \frac{R^{*}}{1-\mu} .
$$

Учитывая равенства (34), получим из этого неравенства оценки для $\varepsilon_{i}$

$$
\left|\varepsilon_{i}\right| \leqslant \tau_{i}+h^{n} \sum_{k=\bar{p}}^{\bar{q}}\left|g_{i k}\right| \cdot \frac{R^{*}}{1-\mu} \quad(i=p, p+1, \ldots, q) .
$$

Такой метод оценки погрешности применим и в случае уравнений более высокого порядка, а также в случае более точных многоточечных методов.

\section{4. Обыкновенный метод конечных разностей решения краевых задач для системы уравнений четного порядка}

Метод оценки погрешности, изложенный в статье [3], можно приспособить и для оценки погрешности обыкновенного разностного метода решения системы линейных дифференциальных уравнений. Мы будем рассматривать только краевые задачи для системы уравнений второго порядка при краевых условиях, из которых каждое содержит значения только одной неизвестной функции. Обобщение получаемых результатов на случай краевых задач для системы уравнений произвольного четного порядка не представляет трудностей, если каждое из краевых условий содержит только одну неизвестную функцию.

Если хоть одно краевое условие содержит значения нескольких неизвестных функций или если порядок уравнений - нечетное число, то проблема оценки погрешности значительно усложняется.

Итак, рассмотрим краевую задачу*

$$
\begin{aligned}
& x_{j}^{\prime \prime}+\sum_{k=1}^{n}\left[q_{i k}(s) x_{k}^{\prime}+p_{i k}(s) x_{k}\right]=f_{j}(s), \\
& a_{j 0} x_{j}(a)-a_{j 1} x_{j}^{\prime}(a)=\alpha_{j}, \\
& b_{j 0} x_{j}(b)+b_{j 1} x_{j}^{\prime}(b)=\beta_{j},
\end{aligned}
$$

где $a_{i i}$ и $b_{j i}(i=0,1)$ - некоторые неотрицательные постоянные.

Для приближенного решения этой краевой задачи применим обыкновенный разностный метод

* Здесь и в дальнейшем $j=1,2, \ldots, n$. Решения этой краевой задачи обозначим через $x_{j}^{*}(s)$.

2 ENSV TA Toimetised F-2 65. 


$$
\left.\begin{array}{c}
\frac{x_{j, i+1}-2 x_{j i}+x_{i, i-1}}{h^{2}} \sum_{k=1}^{n}\left[q_{j k i} \frac{x_{k, i+1}-x_{k, i-1}}{2 h}+p_{j k i} x_{k i}\right]=f_{i i} \\
a_{j 0} x_{j 0}-a_{j 1} \frac{x_{i 1}-x_{j,-1}}{2 h}=\alpha_{j}, \\
b_{j 0} x_{j m}+b_{j 1} \frac{x_{i, m+1}-x_{j, m-1}}{2 h}=\beta_{j},
\end{array}\right\}
$$

где

$$
q_{i k i}=q_{i k}\left(s_{i}\right), p_{j k i}=p_{i k}\left(s_{i}\right), f_{i i}=f_{j}\left(s_{i}\right) .
$$

Способ получения оценки погрешности этого разностного метода вполне аналогичен способу, использованному в статье [3]. Поэтому мы приводим оценку погрешности без доказательства.

Пусть $g_{j k i}$ являются элементами дискретной функции Грина задачи *

$$
u_{j, i+1}-2 u_{i i}+u_{j, i-1}=0 \quad(i=0,1, \ldots, m)
$$

при условиях

$$
\left.\begin{array}{l}
a_{j 0} u_{i 0}-a_{j 1} \frac{u_{i 1}-u_{j,-1}}{2 h}=0, \\
b_{j 0} u_{j m}+b_{j 1} \frac{u_{j, m+1}-u_{j, m-1}}{2 h}=0
\end{array}\right\}
$$

и $t_{j i}=e_{j 0}+e_{j 1} i$ удовлетворяют условиям

$$
\begin{aligned}
& a_{j 0} t_{j 0}-a_{j 1} \frac{t_{j 1}-t_{j,-1}}{2 h}=-a_{j 1} \frac{h^{2}}{6} x_{j}^{* \prime \prime \prime}\left(\xi_{j 0}^{(3)}\right), \\
& \dot{b}_{j 0} t_{j m}+b_{j 1} \frac{t_{j, m+1}-t_{j, m-1}}{2 h}=b_{j 1} \frac{h^{2}}{6} x_{j}^{* \prime \prime \prime}\left(\xi_{j m}^{(3)}\right) .
\end{aligned}
$$

Обозначим

$$
\begin{aligned}
& R_{j i}=\sum_{k=1}^{n}\left[q_{j k i} \frac{t_{k, i+1}-t_{k, i-1}}{2 h}+p_{j k i} t_{k i}\right]+ \\
& +\frac{h^{2}}{12} x_{j}^{* I V}\left(\xi_{j i}^{(4)}\right)+\sum_{k=1}^{n} q_{j k i} \frac{h^{2}}{6} x_{k}^{* \prime \prime \prime}\left(\xi_{k i}^{(3)}\right) \quad\left(s_{i-1} \leqslant \xi_{j i}^{(4)} \leqslant s_{i+1}\right)
\end{aligned}
$$

и оценки

$$
\begin{aligned}
& \max _{\substack{i=0,1, \ldots, m \\
j=1,2, \ldots, n}}\left|p_{j k i}\right| \leqslant \pi_{k 0}, \quad \max _{\substack{i=0,1, \ldots, m \\
j=1,2, \ldots, n}}\left|q_{j k i}\right| \leqslant \pi_{k 1}, \\
& \max _{\substack{i=0,1, \ldots, m \\
l=1,2, \ldots, n}}\left|R_{j i}\right| \leqslant R^{*}, \quad \max _{i=0,1, \ldots, m} h^{2} \sum_{l=0}^{m}\left|g_{k i l}\right| \leqslant \mu_{k 0},
\end{aligned}
$$

* Отметим, что элементы $g_{i k}$ задачи вида $\{(37),(38)\}$ даны в статье [5]. 


$$
\begin{array}{r}
\max _{i=0,1, \ldots, m} h^{2} \sum_{l=0}^{m} \frac{\left|g_{k, i+1, l}-g_{k, i-1, l}\right|}{2 h} \leqslant \mu_{k 1}, \quad\left|t_{j i}\right| \leqslant \tau_{j i} \\
(i=0,1, \ldots, m) .
\end{array}
$$

Те орем а 2. Ecлu

$$
\mu=\sum_{k=1}^{n}\left(\pi_{k 1} \mu_{k 1}+\pi_{k 0} \mu_{k 0}\right)<1,
$$

то система (36) имеет единственное решение $\left\{x_{j i}\right\}$, причем имеет место оценка, погрешности

$$
\begin{aligned}
&\left|x_{j}^{*}\left(s_{i}\right)-x_{j i}\right| \leqslant \tau_{j i}+h^{2} \sum_{k=0}^{m}\left|g_{j i k}\right| \frac{R^{*}}{1-\mu} \\
&(i=1,2, \ldots, m ; \quad i=-1,0,1, \ldots, m+1) .
\end{aligned}
$$

\section{Л ИТЕРАТ У РА}

1. Березин И. С., Ж идков Н. П., Методы вычислений, II, М., 1959.

2. Колл а ц Л., Численные методы решения дифференциальных уравнений, М., 1953,

3. Ю рген с он Р., Изв АН ЭССР. Сер. физ-матем. и техн. наук, № 4, 391 (1963).

4. Ю р ге н сон Р., Тр. Ин-та физ. и астрон. АН ЭССР, 24, 36 (1964).

5. Юр ген сон Р., Тр. Ин-та физ. и астрон. АН ЭССР, 24, 25 (1964).

Институт физики и астрономии

Академии наук Эстонской ССР

\section{Поступила в редакцию} 22/VII 1964

\section{R. JURGENSON}

\section{MONEDE DIFERENTSMEETODITE VEAHINNANGUST HARILIKE LINEAARSETE DIFERENTSIAALVORRANDITE LAHENDAMISEL}

Käesolevas artiklis vaadeldakse artiklis $\left[{ }^{3}\right]$ saadud üldiste veahinnangute rakendamist mōnede konkreetsete diferentsmeetodite korral.

\section{R. JORGENSON}

\section{ON THE ERROR ESTIMATE OF FINITE DIFFERENCE METHODS APPLIED FOR USUAL LINEAR DIFFERENTIAL EQUATIONS}

In this paper some applications of the general error estimates found in the paper [ [ 1 for some concrete finite difference methods are considered. 